\title{
Identificação de Modelos MISO para Geração de Energia Solar Fotovoltaica Usando Redes Neurais Artificiais
}

\author{
Ronnyel C. C. Silva*. José M. M. Júnior** \\ *Curso de Engenharia Elétrica, Universidade Federal do Piauí, Campus Universitário Ministro Petrônio Portella, s/n Bairro
Ininga -Teresina - PI CEP: 64049-550 (e-mail: ronnyelcarlos@ gmail.com). \\ **Curso de Engenharia Elétrica, Universidade Federal do Piauí, Campus Universitário Ministro Petrônio Portella, s/n \\ Bairro Ininga·Teresina - PI CEP: 64049-550 (e-mail: josemenezesjr@ufpi.edu.br).
}

\begin{abstract}
The use of alternative sources of energy has been growing in recent years, in this context, photovoltaic solar energy has a great presence in this market, having been a great source of world energy. Consequently, new methods of identifying models of photovoltaic solar generation need to be implemented in search of an adequate energy program. Artificial neural networks have been used for computational solar incidents based on meteorological and environmental parameters. However, the use of Multiple Input Single Output (MISO) models for photovoltaic generation has been little explored by the researchers. In this work I use artificial neural networks with multiple linear layers and regression to estimate a generation of photovoltaic solar energy for a photovoltaic system to a series of variables of direct horizontal solar radiation, wind velocity and ambient temperature.
\end{abstract}

Resumo: O uso de fontes alternativas de energia vem crescendo consideravelmente nos últimos anos, neste âmbito, a energia solar fotovoltaica tem apresentado grande participação nesse mercado, aumentado a sua participação na matriz energética mundial. Consequentemente, novos métodos de identificação de modelos de geração solar fotovoltaica precisam ser implementados em busca de um planejamento energético adequado. Nesse sentido, redes neurais artificiais têm sido usadas para calcular a radiação solar incidente baseado em parâmetros meteorológicos e ambientais. Entretanto, a utilização de modelos Multiple Input Single Output (MISO) para geração em sistemas fotovoltaicos tem sido pouco explorada pelos pesquisadores. Neste trabalho utilizou-se redes neurais artificiais de múltiplas camadas e regressão linear múltipla para estimar a geração solar fotovoltaica para um sistema fotovoltaico conectado à rede elétrica baseado em variáveis de radiação solar direta horizontal, velocidade do vento e temperatura ambiente.

Keywords: Artificial neural networks; multiple linear regression; solar photovoltaic generation.

Palavras-chaves: Redes neurais artificiais; regressão linear múltipla; geração solar fotovoltaica.

\section{INTRODUÇÃO}

É evidente que o crescimento tecnológico da sociedade como um todo é acompanhado por uma demanda crescente de energia elétrica para o suprimento das necessidades básicas. Tendo em vista esse crescimento e o esgotamento das fontes convencionais não renováveis, torna-se necessário a intensificação do estudo de fontes alternativas de energia elétrica, bem como o desenvolvimento de novas tecnologias para geração de energia.

O uso de fontes alternativas de energia vem crescendo de forma considerável nos últimos anos, e neste cenário, a energia solar fotovoltaica tem sido uma forte representante. Embora seja a energia eólica a maior detentora do volume de investimentos, o Brasil, em virtude da sua localização - na maior parte na região intertropical - possui grande potencial para que haja o aproveitamento de energia solar durante todo o ano. Além disso, a utilização desta forma de energia contribui a longo prazo para o país, já que a taxa de retorno de investimento não é alta em relação ao custo de eletrificação pela rede convencional em regiões remotas (Pereira et al., 2008).

Outro fator que contribui para a utilização da energia solar em detrimento da energia convencional é o esgotamento da capacidade de ampliação de geração hidráulica, além do intenso uso das termelétricas, que acabam tornando o preço da energia elétrica cada vez maior (EPE, 2018). Mesmo em decorrência da localização estratégica do Brasil, a utilização da energia solar não se restringe ao contexto nacional, sendo a China e os Estados Unidos os maiores líderes em geração de energia solar no mundo (Xiangwen, 2012). 
A geração de energia solar fotovoltaica ocorre por meio da utilização de estruturas contendo um conjunto de células fotovoltaicas, recebendo o nome de painel fotovoltaico, que faz uso do efeito fotovoltaico, convertendo luz em eletricidade (Panwar et al, 2011).

A energia proveniente do Sol que incide sobre o planeta Terra sob a forma de irradiação solar é responsável, direta ou indiretamente, por quase todas as outras fontes de energia. De forma direta, além de utilizada para o aquecimento de fluidos para geração de potência mecânica, tal energia pode ser convertida em elétrica através de materiais fotovoltaicos, sendo essa uma forma não poluente de gerar eletricidade (De Energia Solar, 2013).

No âmbito do planejamento energético, o conhecimento da geração de energia futura é de suma importância na determinação de medidas a curto, médio e longo prazo para que não seja interrompido o suprimento de energia elétrica. Assim, uma abordagem alternativa na determinação da geração futura em sistemas fotovoltaicos é a utilização de variáveis ambientais, como: radiação horizontal, radiação difusa e temperatura ambiente, como variáveis preditoras (Hashimura, 2012).

Após situar o leitor sobre a relevância do tema estudado, é necessário mostra-lo como o restante deste artigo está estruturado: na seção 2 insere-se a introdução de conceitos básicos e da revisão bibliográfica de trabalhos relacionados. Já na seção 3 é mostrado o desenvolvimento das duas técnicas propostas. Na seção 4 é descrita a metodologia utilizada na realização deste trabalho. Já na seção 5 são apresentados os resultados onde são avaliados os dois modelos utilizados. Na seção 6 são apresentadas as considerações finais, limitações das propostas, bem como um direcionamento para trabalhos futuros.

\section{TRABALHOS RELACIONADOS}

No que tange a geração e consumo de energia elétrica, tem-se que toda a energia consumida é gerada instantaneamente. Portanto, para que toda a demanda de energia seja atendida, instantaneamente, a energia consumida deve ser exatamente igual a energia gerada. Assim, a confiabilidade e estabilidade do sistema elétrico de potência depende diretamente do potencial de geração disponível. Por essas razões, a capacidade de predizer valores futuros de geração solar fotovoltaica é de fundamental importância para o planejamento energético (Lacchini et al., 2016).

Nesse sentido, a utilização de redes neurais artificiais para a predição de valores futuros de geração de energia baseado em dados ambientais de entrada tem proporcionado bons resultados, como os que são apresentados em Muhammad el al., (2014). Jimenez et al., (2016) utiliza redes neurais de base radial (RBF) e redes neurais percetron múltiplas camadas (MLP) para predição da radiação solar global (GSR). O modelo utilizado usa como variáveis de entrada a velocidade do vento, a humidade relativa do ar e os meses do ano.

Rizwan et al., (2012) aplica redes neurais MLP e lógica fuzzy para predizer valores futuros de radiação solar global (GSR). O modelo utilizado pelos autores usava como parâmetros os dados de latitude, longitude, altitude, horas de sol, temperatura ambiente e meses do ano. Guarnieri (2006) aplica redes neurais MLP e regressão linear múltipla para predição de GSR no modelo regional ETA (modelo de previsão de variáveis meteorológicas) aplicado a estações meteorológicas.

Chatziagorakis et.al., (2014) utiliza redes neurais treinadas a partir do algoritmo Levenberg-Marquardt para melhorar a gestão de energia através da variável de irradiação solar do ambiente. Pinheiro et al., (2017) utiliza redes neurais treinadas a partir do algoritmo Levenberg-Marquard para previsão da energia elétrica futura gerada por um sistema fotovoltaico conectado à rede elétrica.

Kumar et al., (2018) utiliza um modelo de circuito equivalente a um único diodo para a simulação detalhada passo a passo de um módulo fotovoltaico solar sob o ambiente Matlab / Simulink e analisa o desempenho do módulo simulado mediante variação de parâmetros meteorológicos.

Hassan et al., (2017) utiliza um modelo matemático para modelar os componentes de radiação solar e a geração de energia de painéis fotovoltaicos. Onde mostra-se que o modelo de predição pode ser usado na detecção de falhas do sistema fotovoltaico.

Saberian et al., (2014) aplicou um método de modelagem de energia solar utilizando redes neurais artificiais. Duas estruturas de redes neurais foram utilizadas para modelar a potência de saída de um painel solar fotovoltaico, a saber uma rede de propagação feedforward (FFBP) e uma rede de regressão geral (GRNN).

Portanto, observou-se que a maioria dos estudos realizados, que se dedicam a tarefa de previsão de geração de energia solar fotovoltaica relacionam-se ao dimensionamento de inversores de frequência e à previsão da radiação da radiação solar. Com exceção de Pinheiro et al., (2017) nenhum dos demais pesquisadores utilizaram redes neurais em seus trabalhos para predizer a geração solar fotovoltaica de um sistema solar fotovoltaico.

Assim, este artigo apresenta como proposta diferente das demais a aplicação de dois métodos para a identificação de modelos MISO (Multiple Input Single Output) para geração de energia solar fotovoltaica utilizando redes neurais artificiais e regressão linear múltipla.

\section{FUNDAMENTAÇÃO TEÓRICA}

\subsection{Regressão Linear Múltipla Aplicada a Previsão de Geração Solar Fotovoltaica}

Propomos inicialmente um modelo de regressão linear múltipla em que Y representa a geração solar fotovoltaica, V.V a velocidade do vento, T.A expressa a temperatura ambiente, R.D.H é a radiação direta horizontal, conforme mostra a Fig. 1 .

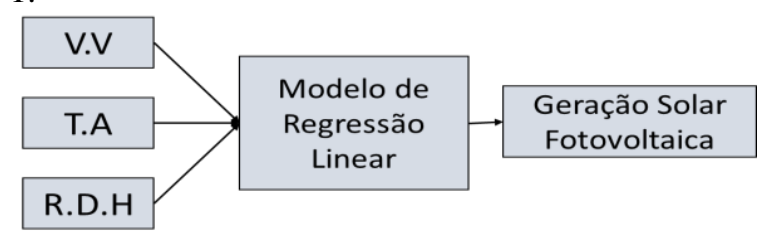

Fig. 1 Modelo usando regressão linear múltipla 
Portanto, o modelo mostrado na Fig. 1 tem a saída estimada conforme é visto na equação 1 :

$$
Y=\beta o+\beta_{1} x_{1}+\beta_{2} x_{2}+\cdots+\beta_{k} x_{k}+e .
$$

Onde:

$$
\begin{aligned}
& \beta i \text { : são os coeficientes de regressão real; } \\
& x i \text { : são os parâmetros de entrada do modelo; } \\
& e \text { : é o erro do modelo. }
\end{aligned}
$$

\subsection{Redes Neurais Artificiais de Múltiplas Camadas Aplicada a Previsão de Geração Solar Fotovoltaica}

A segunda abordagem adotada nesse trabalho para a quantificação da geração de energia elétrica de sistemas fotovoltaicos a partir das variáveis ambientais é feita utilizando a rede neural de múltiplas camadas (MLP).

Por razões explicadas em tópicos anteriores, utilizou-se como entradas da rede neural a velocidade do vento, a temperatura ambiente e a radiação direta horizontal. Já a saída foi definida como sendo a geração de energia solar fotovoltaica, conforme mostra a Fig. 2.

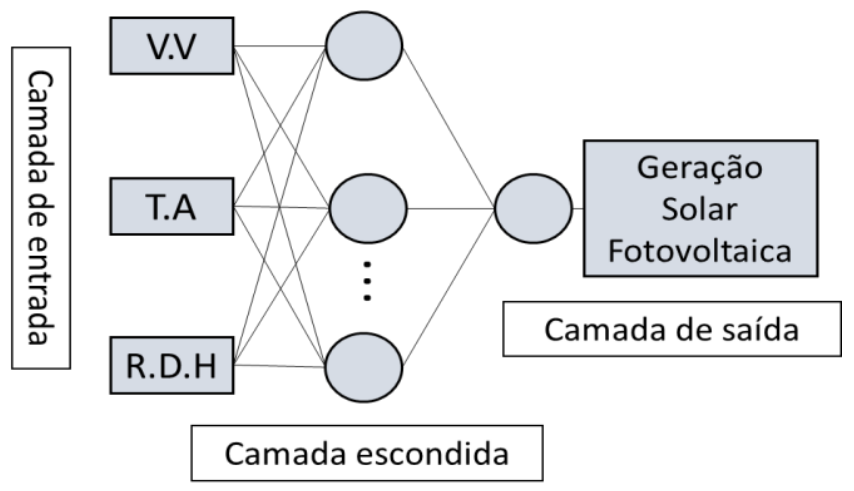

Fig. 2 Modelo usando a rede MLP.

\section{METODOLOGIA}

Neste trabalho foram utilizados dois bancos de dados fornecidos pela plataforma Open Power System Data: banco de dados climatológicos e banco de dados de geração fotovoltaica de sistemas conectados à rede. Para realizar o processamento dos dados e implementação das técnicas que foram aplicadas utilizou-se o software Matlab ${ }^{\circledR}$ (Mathworks, 2015). Os dados se referem a um sistema de geração fotovoltaico instalado na Alemanha e o intervalo de tempo dos dados utilizado estendeu-se pelo período de 1 ano, em intervalos de uma hora, referente ao ano de 2016, nos quais os seguintes dados encontram-se disponíveis:
I) temperatura ambiente;
II) velocidade do vento;
III) radiação direta horizontal;
IV) radiação difusa horizontal;
V) geração solar fotovoltaica.

Para a montagem dos padrões de entrada e posterior alimentação dos modelos foi necessário realizar uma análise inicial nas duas bases de dados (base climatológica e base de geração solar fotovoltaica), buscando por incoerências específicas presentes na mesma.

Nessa etapa fez-se uma busca na base de dados por pontos "Outliers"e "NaN", ou seja, que não fazem parte da distribuição entre as variáveis do problema abordado. Após a etapa de pré-processamento dos dados, calculou-se a correlação entre as variáveis climatológicas e a variável de saída para identificar as variáveis que seriam utilizadas como entrada dos modelos computacionais, conforme mostra a Tabela 1.

Tabela 1. Correlação entre as variáveis climatológicas e a geração solar fotovoltaica

\begin{tabular}{|c|c|}
\hline Entrada & $\begin{array}{c}\text { Geração } \\
\text { fotovoltaica }\end{array}$ \\
\hline Velocidade do vento & 0,1018 \\
\hline Temperatura ambiente & 0,5612 \\
\hline $\begin{array}{c}\text { Radiação direta } \\
\text { horizontal }\end{array}$ & 0,9132 \\
\hline $\begin{array}{c}\text { Radiação difusa } \\
\text { horizontal }\end{array}$ & 0,7442 \\
\hline
\end{tabular}

Por seguinte, elaborou-se a Tabela 2 que possui a correlação entre os atributos de entrada dos modelos. Nota-se que a variável de radiação solar difusa horizontal possui alta correlação com a variável de radiação solar direta horizontal $(0,78)$ e por essa razão ela foi desconsiderada como variável de entrada dos modelos, tendo em vista que a alta correlação entre as variáveis de entrada dificulta o processo de identificação de causa e efeito dos atributos de entrada em relação a saída do modelo (Aguirre, 2007).

Tabela 2. Correlação entre as variáveis climatológicas de entrada dos modelos.

\begin{tabular}{|c|c|c|c|c|}
\hline & $\begin{array}{c}\text { Velocida } \\
\text { de } \\
\text { do vento }\end{array}$ & $\begin{array}{c}\text { Temperatura } \\
\text { ambiente }\end{array}$ & $\begin{array}{c}\text { Radiação } \\
\text { difusa } \\
\text { horizontal }\end{array}$ & $\begin{array}{c}\text { Radiação } \\
\text { direta } \\
\text { horizontal }\end{array}$ \\
\hline $\begin{array}{c}\text { Velocida } \\
\text { de do } \\
\text { vento }\end{array}$ & 1 & $-0,1317$ & 0,0567 & 0,1758 \\
\hline $\begin{array}{c}\text { Temperatu } \\
\text { ra } \\
\text { ambiente }\end{array}$ & $-0,1317$ & 1 & 0,5252 & 0,4666 \\
\hline $\begin{array}{c}\text { Radiação } \\
\text { difusa } \\
\text { horizontal }\end{array}$ & 0,0567 & 0,5252 & 1 & 0,7801 \\
\hline $\begin{array}{c}\text { Radiação } \\
\text { direta } \\
\text { horizontal }\end{array}$ & 0,1758 & 0,4666 & 0,7801 & 1 \\
\hline
\end{tabular}

Portanto, as variáveis velocidade do vento, temperatura ambiente e radiação direta horizontal serão utilizadas como entradas para os modelos de regressão linear múltipla e para o modelo de redes neurais artificiais.

\subsection{Métricas de Avaliação dos Modelos}

Outros parâmetros importantes do modelo são os resíduos. O resíduo para a i-ésima observação pode ser definido como uma função do tipo $r_{i}=y_{i}-d_{i}$ que procura medir a discrepância 
entre o valor real e o valor calculado da i-ésima observação. O sinal de $r_{i}$ indica a direção dessa discrepância.

Além dos resíduos, outra forma de avaliar a qualidade do ajuste do modelo na tarefa de regressão é através do coeficiente de determinação. Basicamente, tal coeficiente indica quanto o modelo foi capaz de adaptar-se aos dados coletados. O coeficiente de determinação é dado pela equação 2:

$$
R^{2}=\frac{\sum_{i=0}^{n}(D i-\widehat{\bar{y} l})^{2}}{\sum_{i=0}^{n}(D i-\widehat{y \imath})^{2}}
$$

Onde:

Di é a i-ésima saída desejada;

$\widehat{\bar{y}}$ é a média das observações;

$\widehat{y \iota}$ é o valor estimado para a i-ésima saída.

Portanto, o coeficiente de determinação é definido como a razão entre a soma dos quadrados da diferença entre a saída desejada e a média das observações e soma dos quadrados da diferença entre os valores desejados e os valores estimados.

O desempenho na identificação dos modelos pode ser quantificado por meio do RMSE (Root Mean Square Error) e do MAE (Mean Absolute Error). Conforme as equações 3 e 4:

$$
\begin{gathered}
R M S E=\sqrt{\frac{1}{n} \sum_{n=0}^{\infty}(D i-\widehat{y \imath})^{2}}, \\
M A E=\frac{1}{n} \sum_{n=0}^{\infty}(D i-\widehat{y \imath}) .
\end{gathered}
$$

\section{RESULTADOS}

Para a obtenção dos resultados de previsão de geração, utilizou-se um banco de dados com 1950 amostras de padrões de entrada e saída, e dividiu-se os dados em uma sub amostra de treinamento, correspondendo a $80 \%$ dos dados, e outra sub amostra contendo $20 \%$ dos dados, correspondendo aos dados de teste do modelo.

\subsection{Regressão Linear Múltipla}

A aplicação do modelo de regressão linear múltipla apresentou como resultados, após a etapa de treinamento, as métricas de avaliação mostradas na Tabela 3:

Após a etapa de treinamento, na etapa de teste do modelo, pode-se observar na Fig. 3 os gráficos da geração calculada e da geração esperada (eixo vertical) para cada amostra de teste (eixo horizontal).

Tabela 3 - Desempenho do modelo de regressão multilinear

\begin{tabular}{|c|c|c|}
\hline \multicolumn{3}{|c|}{ Regressão Multilinear } \\
\hline $\mathrm{R}^{2}$ & MAE $(\mathrm{kWh})$ & RMSE $(\mathrm{kWh})$ \\
\hline 0,81 & 0,0888 & 0,10723 \\
\hline
\end{tabular}

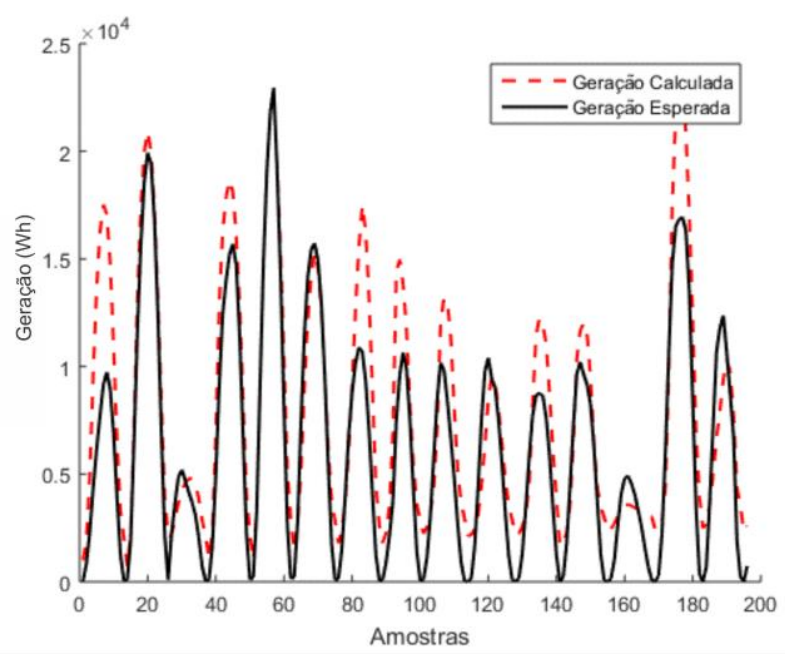

Fig. 3 Geração calculada versus geração esperada para o modelo de regressão Multilinear.

O desempenho do modelo utilizando regressão linear múltipla pode ser verificado mediante a avaliação do gráfico de distribuição de probabilidade do resíduo. Conforme mostra a Fig. 4, a probabilidade do resíduo tende a uma reta, o que indica o seu comportamento aproximadamente normal. Assim, pode-se abstrair que o resíduo tem informação não correlacionada com as amostras de saída do modelo. Portanto, tal gráfico evidencia que o modelo adotado foi capaz de se adapta à maioria dos dados de validação.

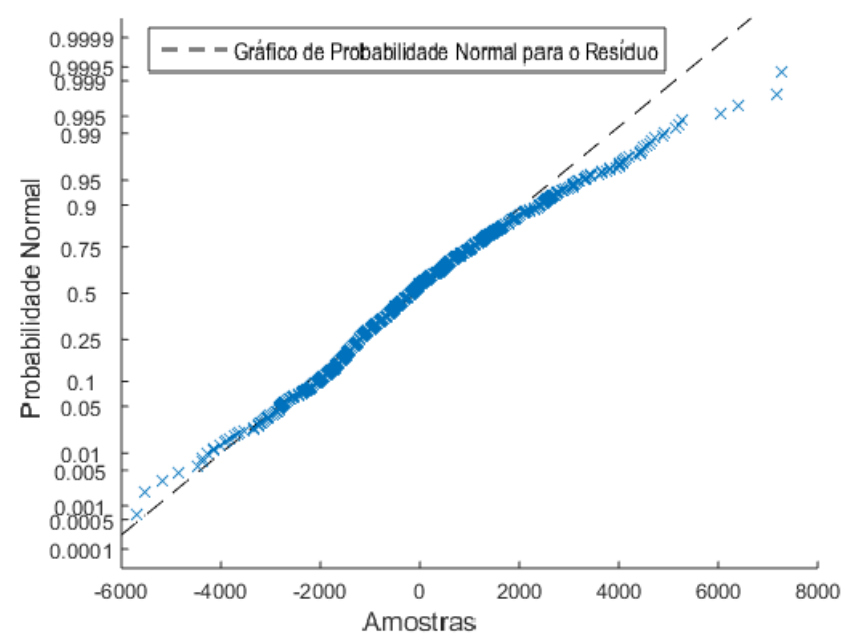

Fig. 4 Probabilidade do resíduo para o modelo de regressão Multilinear.

A Fig. 5 mostra o histograma para os resíduos do modelo de regressão linear múltipla que se aproxima de uma distribuição gaussiana normal, o que evidência, mais uma vez, a adaptação do modelo aos dados de validação. 


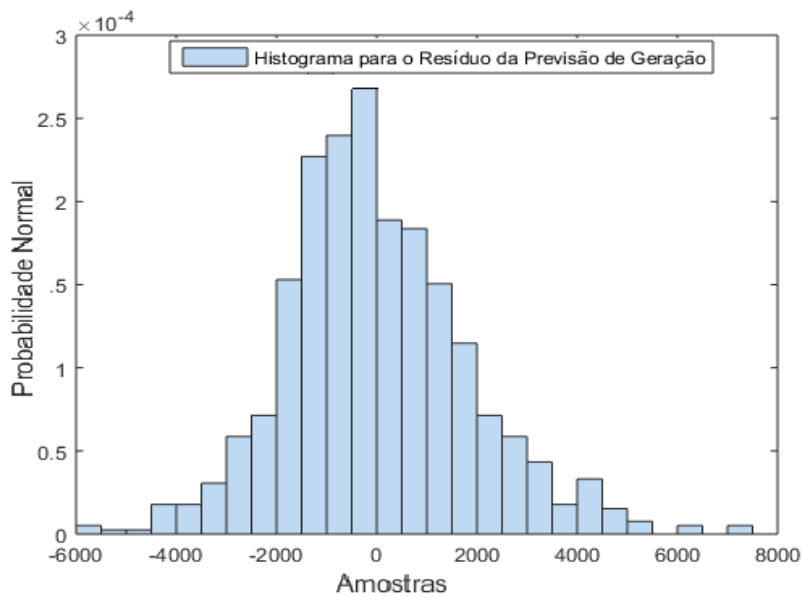

Fig. 5 Histograma do resíduo para o modelo de regressão linear múltipla.

\subsection{Modelo Utilizando a Rede MLP}

A rede neural utilizada foi uma perceptron de múltiplas camadas (MLP) com uma camada de entrada, uma camada escondida e uma camada de saída. Três topologias candidatas foram implementadas a fim de identificar aquela que melhor se adapta ao problema proposto:

a) Uma camada de entrada com três variáveis de entrada, uma camada escondida com cinco neurônios e uma camada de saída com um neurônio (3:5:1);

b) Uma camada de entrada com três variáveis de entrada, uma camada escondida com quinze neurônios e uma camada de saída com um neurônio (3:15:1);

c) Uma camada de entrada com três variáveis de entrada, uma camada escondida com vinte e três neurônios e uma camada de saída com um neurônio (3:23:1).

Para fins de reprodutibilidade, a Tabela 4 evidencia a parametrização das redes neurais implementadas.

Tabela 4 - Parâmetros de configuração da MLP

\begin{tabular}{|c|c|}
\hline \multicolumn{2}{|c|}{ Parâmetros utilizados na MLP } \\
\hline Termo de momentum & 0,8 \\
\hline Taxa de aprendizagem & 0,086 \\
\hline Função de ativação & Sigmoide \\
\hline Épocas & 2000 \\
\hline Repetições & 20 vezes \\
\hline
\end{tabular}

A rede MLP apresentou, para as três topologias implementadas, as métricas de erros mostrados na Tabela 5.

Tabela 5 - Métricas de desempenho do modelo utilizando MLP

\begin{tabular}{|c|c|c|c|c|}
\hline \multirow{2}{*}{$\begin{array}{c}\text { Topologia } \\
\text { da Rede }\end{array}$} & \multicolumn{2}{|c|}{ Treinamento } & \multicolumn{2}{c|}{ Teste } \\
\cline { 2 - 5 } & $\begin{array}{c}\text { MAE } \\
(\mathrm{kWh})\end{array}$ & $\begin{array}{c}\text { MSE } \\
(\mathrm{kWh})\end{array}$ & $\begin{array}{c}\text { MAE } \\
(\mathrm{kWh})\end{array}$ & $\begin{array}{c}\text { RMSE } \\
(\mathrm{kWh})\end{array}$ \\
\hline $3: 5: 1$ & 0,0223 & 0,0489 & 0,024 & 0,0787 \\
\hline $3: 15: 1$ & 0,0220 & 0,04796 & 0,023 & 0,0781 \\
\hline $3: 23: 1$ & 0,0219 & 0,04796 & 0,0223 & 0,0775 \\
\hline
\end{tabular}

Portanto, conforme mostra a Tabela 5, a topologia de rede neural 3:23:1 apresentou os melhores resultados para o estudo realizado. Tendo em vista que se obteve as menores métricas de erro, tanto na etapa de treinamento como também na etapa de teste da rede neural. Para o modelo regressor utilizando a MLP se pode observar na Fig. 6 os gráficos da geração de energia calculada e a geração esperada. Percebe-se que o modelo regressor utilizando a rede neural MLP com vinte e três neurônios na camada escondida possui um desempenho superior ao modelo que utiliza regressão linear múltipla mostrado na Fig. 3.

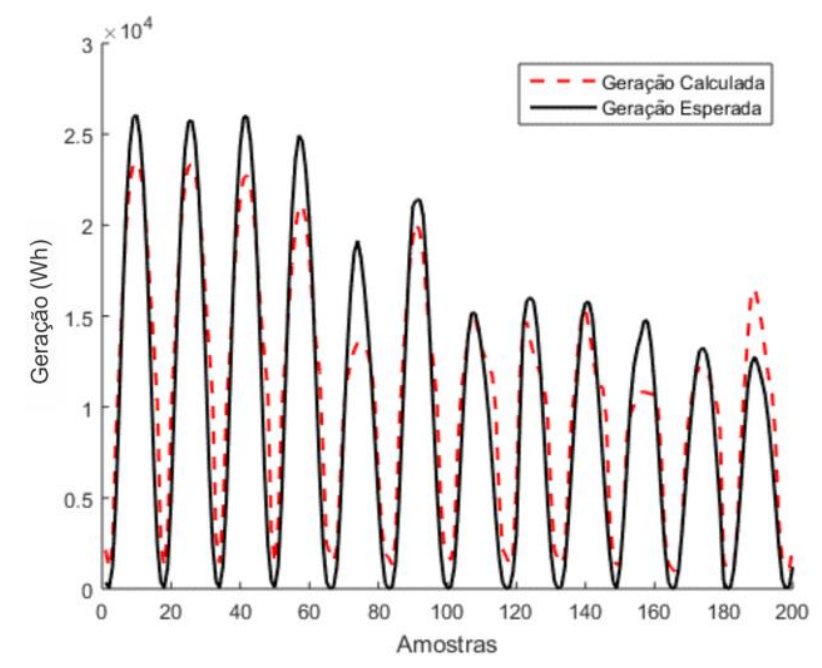

Fig. 6 Geração calculada versus Geração Esperada para o modelo MLP.

Na Fig. 8, tem-se o gráfico de probabilidade do resíduo para o regressor utilizando a rede MLP com a topologia 3:23:1. A análise do gráfico de probabilidade do resíduo evidencia a característica de adaptação do modelo aos dados de teste.

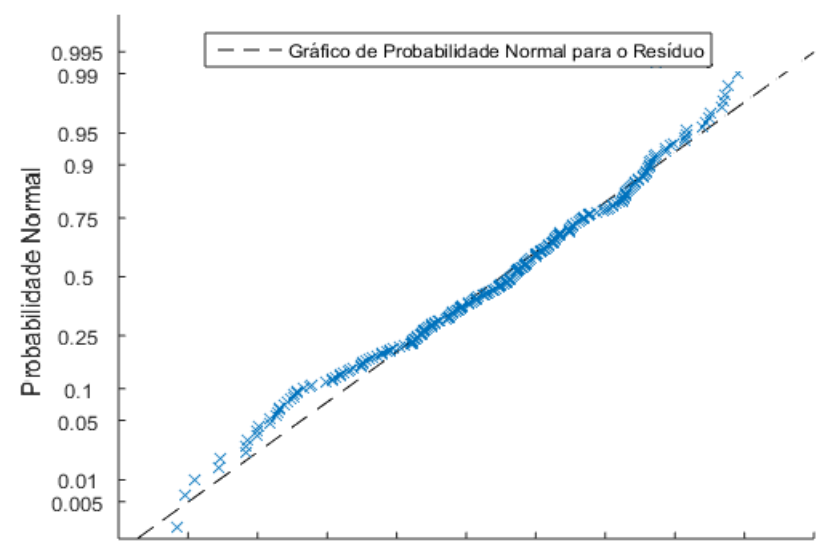

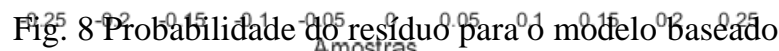
na rede MLP.

A comparação entre as métricas de desempenho do modelo de regressão linear múltipla com o modelo que utiliza a MLP mostra que a rede neural consegue adaptar-se melhor aos parâmetros de entrada, conforme mostra a Tabela 6, 
apresentando valores inferiores para os resíduos, RMSE e MAE.

Tabela 6 - Comparação de desempenho dos dois modelos utilizados

\begin{tabular}{|c|c|c|}
\hline Modelo & MAE $(\mathrm{kWh})$ & RMSE $(\mathrm{kWh})$ \\
\hline $\begin{array}{c}\text { Regressão } \\
\text { Linear Múltipla }\end{array}$ & 0,0888 & 0,1072 \\
\hline MLP & 0,0223 & 0,0775 \\
\hline
\end{tabular}

\section{CONCLUSÕES}

Este trabalho apresentou como contribuições a aplicação de redes neurais artificiais de múltiplas camadas na identificação de modelos MISO para a geração de energia solar fotovoltaica em situações onde se tem o conhecimento apenas das variáveis ambientais da região.

O trabalho permitiu a comparação entre um modelo clássico de identificação por meio da aproximação de funções que utiliza regressão linear múltipla com um modelo regressor utilizando redes neurais perceptron de múltiplas camadas (MLP).

Os resultados do presente trabalho mostram que o modelo utilizando redes neurais de múltiplas camadas possui desempenho superior àquele que utiliza regressão linear múltipla na tarefa de identificação da relação entre a entrada e a saída. Portanto, o modelo usando redes MLP mostrou-se satisfatório na tarefa de identificação da produção de energia de sistemas fotovoltaicos.

\section{AGRADECIMENTOS}

Os autores agradecem ao programa de pós-graduação em Engenharia Elétrica da Universidade Federal do Piauí e ao Grupo de Pesquisa em Automação e Sistemas Inteligentes

\section{REFERÊNCIAS}

Aguirre, Luis Antonio. Introdução à identificação de sistemas-Técnicas lineares e não-lineares aplicadas a sistemas reais. Editora UFMG, 2007.

Chatziagorakis, P.; Elmasides, C.; Sirakoulis, G. C.; et al. Application of Neural Networks Solar Radiation Prediction for Hybrid Renewable Energy Systems. Springer International Publishing Switzerland, , n. 2001, p. 133-144, 2014.

De Energia Solar, Grupo de Trabalho. Fotovoltaica (CRESESB/CEPEL)-. Manual de Engenharia para Sistemas Fotovoltaicos, Disponível em: http://www. cresesb. cepel. br/index. php, 2013.

E. B. Pereira, F.R. Martins, S.A.B. Silva, S.I. Abreu (2008). Solar energy scenarios in Brazil, Part one: Resource assessment, 36(8), 2853 - 2864.

Empresa, de Pesquisa Energética. Balanço energético nacional 2018: ano base 2017. Rio de Janeiro: EPE, 2018.

Guarnieri, Ricardo André. Emprego de redes neurais artificiais e regressão linear múltipla no refinamento das previsões de radiação solar do modelo Eta. Unpublished master's thesis, Instituto Nacional de Pesquisas Espaciais, 2006.
Hashimura, Luís de Medeiros Marques. Aproveitamento do potencial de geração de energia elétrica por fontes renováveis alternativas no brasil: instrumentos de política e indicadores de progresso. unpublished MSc thesis, Universidade Federal do Rio de Janeiro, Instituto Alberto Luiz Coimbra de Pós-Graduação e Pesquisa de Engenharia Energy Planning Program, 2012.

Hassan, Qusay; Jaszczur, Marek; Przenzak, Estera. Mathematical model for the power generation from arbitrarily oriented photovoltaic panel. In: E3S Web of Conferences. EDP Sciences, 2017. p. 01028.

Jimenez, V. A., Barrionuevo, A., Will, A., \& Rodríguez, S. (2016). Neural Network for Estimating Daily Global Solar Radiation Using Temperature, Humidity and Pressure as Unique Climatic Input Variables. Smart Grid and Renewable Energy, 7(03), 94.

Kumar, Raj et al. Solar photovoltaic modeling and simulation: As a renewable energy solution. Energy Reports, v. 4, p. 701-712, 2018.

Lacchini, C.; Pinheiro, E.; Rüther, R. Avaliação de UM Sistema Fotovoltaico Sob a Ótica da. VI Congresso Brasileiro de Energia Solar - Belo Horizonte, 03 a 07 de abril de 2016, v. VI, n. 9788586923432, p. 1-8, 2016.

Matlab e Statistics Toolbox Release 2015b, O MathWorks, Inc., Natick, Massachusetts, Estados Unidos.

Muhammad, E. R, Sishaj P. S., Venkateswaran P. Day-ahead prediction of solar power output for grid-connected solar photovoltaic installations using Artificial Neural Networks. 2014. IEEE 2nd International Conference on Emerging Electronics (ICEE).

Panwar N.L., S.C. Kaushik, S. Kothari (2011). Role of renewable energy in environmental protection: A review. Renewable an Sustainable Energy Reviews, 15(2), $1513-$ 1524.

Pinheiro, Elisangela; Lovato, Adalberto; Ruther, Ricardo. Aplicabilidade de redes neurais artificiais para análise de geração de energia de um sistema fotovoltaico conectado à rede elétrica. Revista Brasileira de Energias renováveis, v. 6, n. $5,2017$.

Rizwan, M.; Jamil, Majid; Kothari, D. P. Generalized neural network approach for global solar energy estimation in India. IEEE Transactions on Sustainable Energy, v. 3, n. 3, p. 576-584, 2012.

Saberian, Aminmohammad et al. Modelling and prediction of photovoltaic power output using artificial neural networks. International journal of Photoenergy, v. 2014, 2014.

Xiangwen, Z., Ran, C., Chun, W, and Xinlong, Z. (2012). Design of Remote Monitoring and Control System Center of Photovoltaic Power Station. IEEE PES Innovative Smart Grid Technologies, pp. 1- 3. 